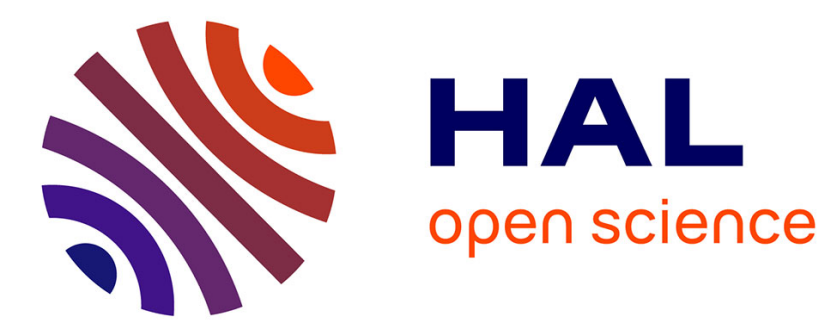

\title{
Actualités du traitement de l'hépatite C
}

Frédéric Moal, Nicolas Terrail

\section{To cite this version:}

Frédéric Moal, Nicolas Terrail. Actualités du traitement de l'hépatite C. Actualités Pharmaceutiques, 2019, 58, pp.27 - 31. 10.1016/j.actpha.2018.11.006 . hal-03486838

\section{HAL Id: hal-03486838 \\ https://hal.science/hal-03486838}

Submitted on 21 Dec 2021

HAL is a multi-disciplinary open access archive for the deposit and dissemination of scientific research documents, whether they are published or not. The documents may come from teaching and research institutions in France or abroad, or from public or private research centers.
L'archive ouverte pluridisciplinaire HAL, est destinée au dépôt et à la diffusion de documents scientifiques de niveau recherche, publiés ou non, émanant des établissements d'enseignement et de recherche français ou étrangers, des laboratoires publics ou privés.

\section{다)(1) $\$$}

Distributed under a Creative Commons Attribution - NonCommerciall 4.0 International 


\title{
Dochead dossier
}

Sous-dochead Hépatite $C$, la révolution en marche

\section{Actualité du traitement de l'hépatite $C$}

\author{
Frédéric Moal ${ }^{\mathrm{a}, \mathrm{b}}$
}

Professeur associé en pharmacie clinique, Pharmacien praticien hospitalier

\section{Nicolas Terrail ${ }^{\mathrm{c}, *}$}

Pharmacien praticien hospitalier

aUniversité d'Angers, 16 boulevard Daviers, 49045 Angers cedex, France

${ }^{\mathrm{b} C e n t r e ~ h o s p i t a l i e r ~ u n i v e r s i t a i r e ~ d ’ A n g e r s, ~} 4$ rue Larrey, 49933 Angers cedex 9, France

${ }^{\mathrm{c} C}$ Centre hospitalier universitaire de Montpellier, Pharmacie Lapeyronie, 371 avenue du DoyenGaston-Giraud, 30295 Montpellier cedex 5, France

\section{*Auteur correspondant.}

Adresse e-mail : n-terrail@chu-montpellier.fr (N. Terrail).

\section{Résumé}

L'apparition de combinaisons d'antiviraux à action directe a métamorphosé la prise en charge de I'hépatite $\mathrm{C}$. Si leur prescription reste hospitalière et réservée aux spécialistes, leur mise à disposition en double circuit pharmaceutique renforce le rôle du pharmacien en matière d'observance, de prévention des effets indésirables et de détection des interactions médicamenteuses. Le remboursement à $100 \%$ favorise l'accès de toutes les populations au traitement.

(C) 2018

Mots clés - antiviral d'action directe ; dispensation pharmaceutique ; interaction médicamenteuse ; virus de l'hépatite $C$

\section{Summary}

New direct-acting antivirals have changed the paradigm of treatment of hepatitis $\mathrm{C}$. If their prescription is still reserved to hospital and specialist physicians, drug availability in both hospital and community pharmacies reinforce the position of the pharmacist for a good observance, counselling 
on side effects and the search for interactions. $100 \%$ reimbursement grants a better access to all populations to treatment.

(c) 2018

Keywords - à venir

Afin d'atteindre l'objectif d'élimination virale de l'hépatite C avant 2025, tel qu'il a été défini par I'Association française pour l'étude du foie (AFEF), plusieurs mesures simultanées sont recommandées autour de deux axes : le traitement et le dépistage universels [1,2].

La mise à disposition des antiviraux d'action directe (AAD) dans les années 2010 a constitué une révolution thérapeutique dans la prise en charge des patients infectés par le virus de I'hépatite C (VHC).

Les molécules les plus récentes sont pangénotypiques, c'est-à-dire qu'elles agissent efficacement quel que soit le génotype viral.

Malgré sa simplicité de prise et sa durée relativement courte (8 ou 12 semaines), le traitement requiert une vigilance extrême du pharmacien, d'officine ou hospitalier, au niveau de la délivrance et du suivi du patient, notamment dans la recherche d'interactions médicamenteuses [1].

\section{T1 Évolution de l’arsenal thérapeutique du VHC}

TEG1 Avant 2010, le traitement de référence de l'hépatite C chronique reposait sur l'association de deux molécules : l'interféron pégylé et la ribavirine. Administré durant 24 à 48 semaines selon le profil (ou génotype) du virus, ce traitement permettait d'atteindre la guérison chez près de $40 \%$ des personnes traitées. Pour les autres patients, la bithérapie n'était pas efficace, difficile à suivre ou mal tolérée. À ces personnes en impasse thérapeutique, aucun autre traitement ne pouvait être proposé.

TEG1 Depuis le début des années 2010, un bouleversement thérapeutique s'est amorcé, avec l'apparition des deux premiers AAD en 2011, actifs sur un seul génotype, le génotype 1 : le télaprévir et le bocéprévir. Associé à la bithérapie de référence, chacun d'eux a permis de relever le taux de guérison des personnes traitées à $70 \%$.

TEG1 Depuis 2014, la mise à disposition de nouveaux AAD pangénotypiques, actifs sur tous les génotypes, permet une simplification du traitement, en dehors de situations particulières. Alors que leur dispensation était initialement réservée à l'hôpital, la plupart des combinaisons d'AAD peuvent désormais bénéficier d'un double circuit et être délivrées en pharmacie d'officine. Ces médicaments sont, de plus, remboursables à $100 \%$.

\section{T1 Molécules recommandées}

TEG1 Des associations fixes d'AAD sont disponibles : elles regroupent un inhibiteur de la protéine NS5A du VHC (lédipasvir, elbasvir, velpatasvir, pibrentasvir), importante dans la réplication de l'acide 
ribonucléique (ARN) et l'assemblage des virions du virus, avec un inhibiteur de la polymérase NS5B (sofosbuvir) et/ou un inhibiteur de la protéase NS3A/4A (grazoprévir, glécaprévir, voxilaprévir) essentielle pour la réplication virale [1] (tableau 1).

TEG1 La base du traitement de l'hépatite $\mathbf{C}$ consiste à associer ces molécules antivirales entre elles - le plus souvent dans un comprimé unique - de manière à obtenir une efficacité importante combinée à une barrière de résistance élevée [1,2].

\section{T1 Mécanisme d'action des AAD recommandés}

TEG1 L'identification et la caractérisation des différentes protéines du cycle de réplication du VHC et de leurs unités fonctionnelles ( $p 7, N S 2, N S 3, N S 4 A, N S 4 B, N S 5 A$ et NS5B) ont constitué une avancée majeure [3,4] (figure 1).

TEG1 Leur reconnaissance en tant que potentielles cibles thérapeutiques a permis le développement des AAD, avec l'avènement de trois groupes de molécules :

- les inhibiteurs de protéase NS3/4A agissant par inhibition directe de la sérine protéase NS3/4A, par fixation sur le site actif sérine de la protéine NS3, bloquant ainsi le clivage protéolytique de la polyprotéine codée du VHC essentiel à la réplication virale (grazoprévir, glécaprévir, voxilaprevir) ;

- les inhibiteurs de la NS5A agissant par inhibition directe de la protéine non structurale NS5A impliquée dans la réplication virale, mais dont le rôle est encore mal connu (lédipasvir, elbasvir, velpatasvir, pibrentasvir);

- les inhibiteurs de la NS5B agissant par inhibition de l'ARN polymérase ARN-dépendante NS5B essentielle pour la réplication du virus. Dans ce groupe, la seule molécule recommandée est le sofosbuvir, un inhibiteur nucléotidique, prodrogue d'un nucléotide, qui subit une métabolisation intracellulaire, forme un analogue de l'uridine triphosphate actif pharmacologiquement, qui est incorporé dans l'ARN viral par la polymérase NS5B et agit comme terminateur de chaîne.

TEG1 La ribavirine n'est plus utilisée que chez les patients présentant une cirrhose, l'option thérapeutique suivante étant recommandée :

- sofosbuvir + velpatasvir + ribavirine pendant douze semaines quand l'atteinte est classifiée ChildPugh B7-9, :

- sofosbuvir + lédipasvir + ribavirine pendant douze semaines ou sofosbuvir + velpatasvir + ribavirine pendant 12 semaines quand elle est classifiée Child-Pugh C10-12.

\section{T1 Principaux effets indésirables des AAD}

TEG1 Le profil de tolérance des différents AAD a été globalement satisfaisant au cours des études cliniques. Environ 70 à $90 \%$ des patients ont rapporté au moins un événement indésirable, avec une fréquence moindre pour les durées de traitement courtes (8 à 12 semaines) et en l'absence de 
ribavirine. La majorité de ces effets indésirables a été d'intensité faible à modérée (grade 1

ou 2) (tableau 1).

Tableau 1. Molécules commercialisées en $2018^{1}$.

\begin{tabular}{|c|c|c|c|c|c|c|c|}
\hline $\mathrm{DCl}$ & $\begin{array}{l}\text { Nom de } \\
\text { spécialité }\end{array}$ & Dosage & Efficacité virologique & $\begin{array}{l}\text { Indicat } \\
\text { ion } \\
\text { AMM }\end{array}$ & Posologie & $\begin{array}{l}\text { Durée de } \\
\text { traitement }\end{array}$ & $\begin{array}{l}\text { Principaux } \\
\text { effets } \\
\text { indésirables }\end{array}$ \\
\hline $\begin{array}{l}\text { Sofosb } \\
\text { uvir } \\
+ \text { velp } \\
\text { atasvir }\end{array}$ & Epclusa $^{\circledR}$ & $\begin{array}{l}\text { Comprimé } \\
\text { pelliculé } \\
\text { dosé à } \\
400 \mathrm{mg} \text { de } \\
\text { sofosbuvir } \\
+100 \mathrm{mg} \text { de } \\
\text { velpatasvir }\end{array}$ & $\begin{array}{l}\text { Efficacité } \\
\text { pangénotypique } \\
\text { supérieure à } 90 \%, \\
\text { avec une durée } \\
\text { de traitement } \\
\text { de } 12 \text { semaines sans } \\
\text { adjonction } \\
\text { de ribavirine pour la } \\
\text { majorité des patients } \\
\text { avec ou sans cirrhose }\end{array}$ & $\begin{array}{l}\text { Traite } \\
\text { ment } \\
\text { de } \\
\text { l'infect } \\
\text { ion } \\
\text { chroni } \\
\text { que } \\
\text { par le } \\
\text { virus } \\
\text { de } \\
\text { I'hépat } \\
\text { ite C } \\
\text { (VHC) } \\
\text { chez } \\
\text { les } \\
\text { adulte } \\
\mathrm{s}\end{array}$ & $\begin{array}{l}\text { Un comprimé } \\
\text { par jour à } \\
\text { prendre, avec } \\
\text { ou sans } \\
\text { nourriture }\end{array}$ & 12 semaines & $\begin{array}{l}\text { Maux de } \\
\text { tête, fatigue, } \\
\text { nausées }\end{array}$ \\
\hline $\begin{array}{l}\text { Glécap } \\
\text { revir } \\
+ \text { pibr } \\
\text { entasv } \\
\text { ir }\end{array}$ & Maviret $^{\circledR}$ & $\begin{array}{l}\text { Comprimé } \\
\text { pelliculé } \\
\text { dosé à } \\
100 \mathrm{mg} \text { de } \\
\text { glécaprévir } \\
+40 \mathrm{mg} \text { de } \\
\text { pibrentasvir }\end{array}$ & $\begin{array}{l}\text { Efficacité virologique } \\
\text { pangénotypique } \\
\text { supérieure à } 90 \% \text {, } \\
\text { avec } 8 \text { à } 12 \text { semaines } \\
\text { de traitement pour la } \\
\text { majorité des patients } \\
\text { avec ou sans cirrhose } \\
\text { (cirrhose compensée } \\
\text { uniquement); } \\
\text { efficacité importante } \\
\text { dans des populations } \\
\text { particulières } \\
\text { (insuffisants rénaux ou } \\
\text { hémodialysés) pour } \\
\text { lesquelles les } \\
\text { alternatives sont } \\
\text { limitées }\end{array}$ & $\begin{array}{l}\text { Traite } \\
\text { ment } \\
\text { de } \\
\text { I'infect } \\
\text { ion } \\
\text { chroni } \\
\text { que } \\
\text { par } \\
\text { le VHC } \\
\text { chez } \\
\text { les } \\
\text { adulte } \\
\text { s }\end{array}$ & $\begin{array}{l}\text { Trois } \\
\text { comprimés } \\
\text { par jour en } \\
\text { une prise, avec } \\
\text { de la } \\
\text { nourriture }\end{array}$ & $\begin{array}{l}\text { Sans cirrhose: } \\
8 \text { semaines } \\
\text { Avec cirrhose : } \\
12 \text { semaines }\end{array}$ & $\begin{array}{l}\text { Maux de } \\
\text { tête, fatigue, } \\
\text { nausées; } \\
\text { profil de } \\
\text { tolérance et } \\
\text { de résistance } \\
\text { satisfaisant, } \\
\text { mais } \\
\text { potentiel } \\
\text { important } \\
\text { d'interaction } \\
\text { s } \\
\text { médicament } \\
\text { euses }\end{array}$ \\
\hline
\end{tabular}




\begin{tabular}{|c|c|c|c|c|c|c|c|}
\hline $\begin{array}{l}\text { Sofosb } \\
\text { uvir } \\
+ \text { velp } \\
\text { atasvir } \\
\text { + voxil } \\
\text { aprévi } \\
\text { r }\end{array}$ & Vosevi $^{\circledR}$ & $\begin{array}{l}\text { Comprimé } \\
\text { pelliculé } \\
\text { dosé à } \\
400 \mathrm{mg} \text { de } \\
\text { sofosbuvir } \\
+100 \mathrm{mg} \text { de } \\
\text { velpatasvir } \\
+100 \mathrm{mg} \text { de } \\
\text { voxilaprévir }\end{array}$ & $\begin{array}{l}\text { Efficacité virologique } \\
\text { pangénotypique } \\
\text { supérieure à } 90 \% \text {, } \\
\text { sans adjonction de } \\
\text { ribavirine, avec une } \\
\text { durée de traitement } \\
\text { de } 8 \text { à } 12 \text { semaines, } \\
\text { sans cirrhose ou avec } \\
\text { cirrhose compensée ; } \\
\text { efficacité démontrée } \\
\text { chez les patients en } \\
\text { échec des antiviraux } \\
\text { d'action directe (AAD) } \\
\text { disponibles } \\
\text { (en particulier } \\
\text { inhibiteurs de la NS5A) } \\
\text { après } 12 \text { semaines et } \\
\text { ce, quel que soit le } \\
\text { génotype viral }\end{array}$ & $\begin{array}{l}\text { Traite } \\
\text { ment } \\
\text { de } \\
\text { l'infect } \\
\text { ion } \\
\text { chroni } \\
\text { que } \\
\text { par } \\
\text { le VHC } \\
\text { chez } \\
\text { les } \\
\text { adulte } \\
\mathrm{s}\end{array}$ & $\begin{array}{l}\text { Un comprimé } \\
\text { par jour, avec } \\
\text { de la } \\
\text { nourriture }\end{array}$ & $\begin{array}{l}\text { Patient naïf } \\
\text { d'AAD sans } \\
\text { cirrhose : } \\
8 \text { semaines } \\
\text { Patient naïf } \\
\text { d'AAD avec une } \\
\text { cirrhose } \\
\text { compensée : } \\
8 \text { semaines si } \\
\text { génotype } 3 \\
\text { Patient } \\
\text { préalablement } \\
\text { traité par AAD } \\
\text { sans cirrhose ou } \\
\text { avec une } \\
\text { cirrhose } \\
\text { compensée : } \\
8 \text { semaines }\end{array}$ & $\begin{array}{l}\text { Maux de } \\
\text { tête, fatigue, } \\
\text { nausées; } \\
\text { risque de } \\
\text { bradycardie } \\
\text { sévère et de } \\
\text { troubles de } \\
\text { la } \\
\text { conduction } \\
\text { lié à } \\
\text { l'association } \\
\text { avec } \\
\text { l'amiodarone }\end{array}$ \\
\hline
\end{tabular}

\begin{tabular}{|c|c|c|c|c|c|c|c|}
\hline $\begin{array}{l}\text { Ledipa } \\
\text { svir } \\
+ \text { sofo } \\
\text { sbuvir }\end{array}$ & Harvoni $^{\circledR}$ & $\begin{array}{l}\text { Comprimé } \\
\text { pelliculé } \\
\text { dosé à } 90 \mathrm{mg} \\
\text { de lédipasvir } \\
+400 \mathrm{mg} \text { de } \\
\text { sofosbuvir }\end{array}$ & $\begin{array}{l}\text { Selon l'AFEF, } \\
\text { association } \\
\text { recommandée } \\
\text { uniquement dans } \\
\text { l'hépatite C chronique } \\
\text { de génotype 1, en } \\
\text { absence de cirrhose, } \\
\text { d'après l'AFEF }\end{array}$ & $\begin{array}{l}\text { Traite } \\
\text { ment } \\
\text { de } \\
\text { l'infect } \\
\text { ion } \\
\text { chroni } \\
\text { que } \\
\text { par } \\
\text { le VHC } \\
\text { chez } \\
\text { les } \\
\text { adulte } \\
\text { s et les } \\
\text { adoles } \\
\text { cents } \\
\text { âgés } \\
\text { de } \\
12 \text { ans } \\
\text { à } \\
\text { moins } \\
\text { de } \\
18 \text { ans }\end{array}$ & $\begin{array}{l}\text { Un comprimé } \\
\text { par jour, avec } \\
\text { ou sans } \\
\text { nourriture }\end{array}$ & $\begin{array}{l}\text { Patients sans } \\
\text { cirrhose : } \\
12 \text { semaines } \\
\text { Patients avec } \\
\text { cirrhose: } \\
24 \text { semaines ou } \\
12 \text { semaines en } \\
\text { cas } \\
\text { d'association à } \\
\text { la ribavirine }\end{array}$ & $\begin{array}{l}\text { Maux de } \\
\text { tête, fatigue, } \\
\text { nausées, } \\
\text { irritabilité, } \\
\text { toux, prurit, } \\
\text { muscles } \\
\text { douloureux, } \\
\text { mal au dos } \\
\text { et aux } \\
\text { articulations }\end{array}$ \\
\hline
\end{tabular}




\begin{tabular}{|c|c|c|c|c|c|c|c|}
\hline $\begin{array}{l}\text { Grazo } \\
\text { previr } \\
+ \text { Elba } \\
\text { svir }\end{array}$ & Zepatier $^{\circledR}$ & $\begin{array}{l}\text { Comprimé } \\
\text { pelliculé } \\
\text { dosé à } 50 \mathrm{mg} \\
\text { de } \\
\text { grazoprevir } \\
+100 \mathrm{mg} \text { de } \\
\text { elbasvir }\end{array}$ & $\begin{array}{l}\text { Efficacité virologique } \\
\text { importante, avec une } \\
\text { durée de traitement } \\
\text { de } 12 \text { à } 16 \text { semaines } \\
\text { ( } \pm \text { ribavirine) pour les } \\
\text { patients sans cirrhose } \\
\text { ou avec cirrhose non } \\
\text { compliquée } \\
\text { Selon la HAS, } \\
\text { l'association fixe } \\
\text { elbasvir-grazoprévir } \\
\text { est une option } \\
\text { thérapeutique pour } \\
\text { une hépatite C } \\
\text { chronique de } \\
\text { génotypes } 1 \text { et 4, sans } \\
\text { ou avec cirrhose } \\
\text { compensée (Child- } \\
\text { Pugh A uniquement) }\end{array}$ & $\begin{array}{l}\text { Traite } \\
\text { ment } \\
\text { de } \\
\text { l'infect } \\
\text { ion } \\
\text { chroni } \\
\text { que } \\
\text { par } \\
\text { le VHC } \\
\text { chez } \\
\text { l'adult } \\
\text { e }\end{array}$ & $\begin{array}{l}\text { Un comprimé } \\
\text { par jour, avec } \\
\text { ou sans } \\
\text { nourriture }\end{array}$ & $\begin{array}{l}\text { Génotype VHC } \\
1 a, 1 b, 4: \\
12 \text { semaines }\end{array}$ & $\begin{array}{l}\text { Maux de } \\
\text { tête, fatigue, } \\
\text { nausées, } \\
\text { diarrhée, } \\
\text { insomnie ; } \\
\text { élévation } \\
\text { transitoire } \\
\text { des } \\
\text { transaminas } \\
\text { es (ASAT et } \\
\text { ALAT) }\end{array}$ \\
\hline
\end{tabular}

${ }^{1}$ Ne sont présentées que les molécules recommandées par la Haute Autorité de santé (HAS) et l'Association française pour l'étude du foie (AFEF).

ALAT : alanine aminotransférase ; AMM : autorisation de mise sur le marché ; ASAT : aspartate aminotransférase; $D C l$ : dénomination commune internationale.

Sur 2 colonnes + marge près de son appel

TEG1 Les effets indésirables les plus fréquemment rapportés ont été la fatigue, les céphalées, l'anémie, les insomnies, les nausées, le prurit et l'asthénie. Des élévations des taux d'alanine transaminase (ALAT) et de la bilirubine ont également été relevées avec les combinaisons comportant un inhibiteur de la protéase NS3/A4. Les anémies et hyperbilirubinémies ont principalement été observées dans les groupes recevant la ribavirine.

TEG1 Des cas d'arythmie cardiaque ou de bradycardie ont été signalés lors de traitements concomitants par sofosbuvir \pm lédipasvir et amiodarone, contre-indiquant l'utilisation des traitements à base de sofosbuvir en association à l'amiodarone. Par ailleurs, quelques cas d’hypertension artérielle pulmonaire ont été rapportés sous sofosbuvir.

TEG1 L'Agence européenne du médicament (EMA) a décidé de réévaluer les AAD du VHC, en date du 17 mars 2016, en raison de deux signaux de tolérance :

- un risque de récidive précoce de carcinomes hépatocellulaires après un traitement curatif chez des patients traités par les AAD ; 
- une possible réactivation du virus de l'hépatite $B$ (antigène $H B s$ positif ou antigène HBs négatif et anti-HBc positif) chez certains malades co-infectés VHB-VHC et traités par AAD de l'hépatite C [5].

\section{T1 Interactions médicamenteuses des AAD}

TEG1 Les propriétés pharmacocinétiques particulières confèrent aux AAD des risques accrus d'interactions médicamenteuses :

- ils ont un métabolisme hépatique intense par les isoenzymes du cytochrome P450 (CYP450), principalement l'isoforme CYP3A4 ;

- ce sont des substrats de nombreux transporteurs (glycoprotéine P [Pgp], Breast Cancer Resistance Protein [BCRP], Organic Anion Transporter Polypeptides [OATP], Organic Cation Transporter [OCT]) ;

- ils ont des effets inhibiteurs sur les CYP et/ou certains transporteurs ;

- ils peuvent donc être à la fois la cible (substrat) ou le prédateur (inhibiteur) d'une interaction médicamenteuse.

TEG1 Le sofosbuvir présente peu d'interactions médicamenteuses et en particulier aucune avec les traitements du virus de l'immunodéficience humaine $(\mathrm{VIH})$, les immunosuppresseurs ou la méthadone. À la suite du signalement de cas d'arythmies cardiaques, I'association sofosbuvir + amiodarone doit être évitée. Les autres AAD possèdent un fort potentiel d'interactions médicamenteuses, notamment avec les inducteurs ou inhibiteurs du cytochrome P450 3A (CYP3A4), limitant leur utilisation concomitante avec de nombreux médicaments, en particulier avec certains traitements du $\mathrm{VIH}^{1}$.

\section{T1 Recommandations de l'AFEF}

L'Association française pour l'étude du foie (AFEF) a rendu publiques ses recommandations pour l'élimination de l'infection par le VHC [1].

\section{T2 Traitement universel}

TEG1 La suppression du caractère obligatoire des réunions de concertation pluridisciplinaire (RCP) n'exclut pas la poursuite d'une collaboration avec les centres experts, les praticiens et les structures en charge des patients.

TEG1 Les RCP doivent cependant être poursuivies dans les cas les plus complexes :

- co-infection avec le VHB ;

- insuffisance rénale avec débit de filtration glomérulaire estimé $<30 \mathrm{~mL} / \mathrm{min} / 1,73 \mathrm{~m}^{2}$ ou dialyse ;

- cirrhose décompensée Child-Pugh B ou C ; 
- transplantation d'organe ;

- carcinome hépatocellulaire ;

- échec d'un précédent traitement par agent antiviral direct.

TEG1 Avant de débuter le traitement, que ce soit dans le cadre d'une prise en charge dans le cadre d'un parcours simplifié ou spécialisé, l'AFEF recommande de :

- rechercher d'éventuelles interactions médicamenteuses ${ }^{2}$;

- se documenter sur l'automédication et l'usage de médecine naturelle (millepertuis, compléments alimentaires...) ;

- s'assurer de l'absence de consommation de pamplemousse ou d'orange sanguine pendant le traitement ;

- insister sur la nécessité d’une observance optimale.

TEG1 Pendant le traitement, l'association préconise de mener un interrogatoire sur l'observance, la tolérance et les interactions médicamenteuses auprès du patient.

TEG1 Douze semaines après l'arrêt du traitement, il est conseillé de réaliser une mesure de la charge virale du VHC.

TEG1 Tous les AAD peuvent être rétrocédés par les pharmacies à usage intérieur (PUI) aux patients traités en ambulatoire et la majorité des associations sont disponibles depuis 2018 en double circuit, donc également en pharmacie d'officine. La prescription de ces médicaments reste pour l'instant hospitalière et réservée aux spécialistes en hépato-gastro-entérologie, médecine interne ou en infectiologie. En mars 2018, l'AFEF s'est cependant prononcé en faveur de la prescription des AAD par l'ensemble des médecins, notamment les médecins généralistes.

TEG1 Dans ce parcours simplifié, deux stratégies équivalentes sont recommandées en première intention : Epclusa $^{\circledR}$ (sofosbuvir + velpatasvir) pendant douze semaines ou Maviret ${ }^{\circledR}$ (glécaprevir + pibrentasvir) pendant huit semaines, avec une efficacité équivalente, proche de $100 \%$ [1].

\section{T2 Traitement de recours après échec}

Les patients en échec d'un premier traitement par antiviraux de première génération - sofosbuvir + ribavirine ; sofosbuvir + siméprevir ou daclatasvir ou lédipasvir ; paritaprévir + ombitasvir \pm dasabuvir - peuvent être traités pendant douze semaines par l'une des associations suivantes : sofosbuvir + velpatasvir + voxilaprevir ; glécaprevir + pibrentasvir ; sofosbuvir + glécaprevir + pibrentasvir.

T2 Recherche de mutation de résistance 
La plupart des schémas thérapeutiques ne nécessitent pas la recherche des mutations de résistance (RAS). Toutefois, dans certains cas, celle-ci peut aider à la prise de décision quant au choix de l'association d'AAD. C'est le cas chez les patients en échec d'un traitement comprenant des AAD anti-NS5A de deuxième génération (elbasvir, pibrentasvir, velpatasvir) pour lesquels les RAS persistent dans le temps.

\section{T1 Conseils aux patients}

TEG1 Une éradication du virus est obtenue avec des schémas thérapeutiques simples chez plus de $90 \%$ des personnes infectées par le VHC, mais la prise régulière du traitement pendant toute la durée prévue est une condition sine qua non de son succès [3].

TEG1 Si l'accès de toutes les personnes infectées à un traitement est actuellement possible, il peut être justifié d'attendre avant de le mettre en œuvre dans certaines situations qu'il convient de discuter avec l'équipe soignante : grossesse en cours, précarité sociale extrême, etc.

TEG1 Il est important de rappeler l'importance des facteurs d'aggravation évitables (alcool, surpoids, certains médicaments, autre hépatite virale), y compris après guérison virale, et d'envisager une vaccination contre l'hépatite B et, chez les voyageurs, contre l'hépatite A.

TEG1 Les co-infections rendent la conduite des traitements plus complexe, en particulier celles par le VHB et surtout le VIH.

TEG1 Il faut éviter de partager brosse à dents, rasoirs, ciseaux et aiguilles, et utiliser des préservatifs en cas de rapports homosexuels masculins.

\section{Points à retenir}

- L'arrivée de nouveaux antiviraux d'action directe (AAD) permet de proposer aux patients infectés par le virus de l'hépatite $C$ un traitement court (8 à 12 semaines) et actif sur tous les génotypes, avec un taux de réussite supérieur à $95 \%$.

- La prescription des AAD est hospitalière, réservée aux spécialistes en hépato-gastro-entérologie, médecine interne ou maladies infectieuses.

- Ces médicaments bénéficient, pour la plupart d'entre eux, d'un double circuit de dispensation pharmaceutique.

- Une réunion de concertation disciplinaire préalable au traitement est réservée aux cas les plus complexes.

- Le rôle du pharmacien est important pour la détection des interactions médicamenteuses. 


\section{T1 Conclusion}

La mise sur le marché des AAD a constitué une véritable révolution pour la prise en charge des patients infectés par le VHC. L'éradication du virus en France avant 2025 est dorénavant envisageable. Bien que très efficaces, ces traitements coûteux requièrent une vigilance accrue en termes de recherche d'interactions médicamenteuses potentielles et de suivi de l'observance.

\section{Notes}

${ }^{1}$ Une description détaillée de ces interactions est disponible dans les résumés des caractéristiques du produit (RCP) et sur le site www.hep-druginteractions.org.

${ }^{2}$ www.hep-druginteractions.org ou application smartphone HEP iChart.

\section{Références}

[1] Association française pour l'étude du foie (AFEF). Recommandations AFEF pour l'élimination de l'infection par le virus de l'hépatite C, en France. Mars 2018. https://afef.asso.fr/wpcontent/uploads/2018/06/VF-INTERACTIF-RECO-VHC-AFEF-v2103.pdf

[2] Haute Autorité de santé (HAS). Rapport d'évaluation des antiviraux d'action directe dans le traitement de l'hépatite $\mathrm{C}$ - Actualisation 2017. www.has-

sante.fr/portail/upload/docs/application/pdf/2018-

03/rapport_actualisation_strat_aad_2017_avis2_06122017_cteval318.pdf

[3] Vidal recos hépatite C. http://hoptimal-p.chu-angers.intra:8012/showReco.html?recold=1746

[4] Thériaque. www.theriaque.org/apps/contenu/accueil.php

[5] Agency EM. EMA reviews direct-acting antivirals for hepatitis C. London: EMA ; 2016.

Déclaration de liens d'intérêts

Les auteurs déclarent ne pas avoir de liens d'intérêts.

Figure

Ter_fig1

Figure 1. Cycle de réplication du virus de l'hépatite $C$ et cibles thérapeutiques potentielles.

(C) N. Terrail

Sur 2 colonnes près de son appel 
